\title{
Micropower Materials Development for Wireless Sensor Networks
}

\author{
Dan Steingart (University of California-Berkeley, USA), Shad Roundy (LV Sensors, Inc., USA), Paul K. \\ Wright (University of California-Berkeley, USA), and James W. Evans (University of California- \\ Berkeley, USA)
}

\section{Microsensors Need Micropower}

Subcentimeter wireless computers capable of interfacing physically with their environment and communicating with each other have progressed from concept ${ }^{1}$ to commercial reality ${ }^{2}$ in the past decade. Wireless sensor nodes are an exciting technology, as they provide a backbone to measure almost any quantity in a spatially disperse way, allowing time-synchronized correlations over meters or miles. Before these devices can be deployed to monitor and protect environments (such as grid power distribution systems, buildings, factories, or even the human body) for long periods of time, they need a power source. Environmental generation looks to be a promising method.

Power requirements of wireless sensor nodes (WSNs) are functions of four main factors: sending, receiving, polling, and sleeping. Table I details the power costs for these steps.

Table I: General Energy Costs of Wireless Sensor Nodes.

\begin{tabular}{|c|c|c|c|}
\hline Function & Power Cost & Time & Factors \\
\hline Transmit & $500 \mu \mathrm{W}-50 \mathrm{~mW}$ & $<0.1 \%$ & $\begin{array}{l}\text { Distance, data } \\
\text { rate }\end{array}$ \\
\hline Receive & $50 \mu \mathrm{W}-10 \mathrm{~mW}$ & $<0.5 \%$ & Signal quality \\
\hline Poll & $5 \mu \mathrm{W}-20 \mathrm{~mW}$ & $<1 \%$ & $\begin{array}{l}\text { Data/actuation } \\
\text { required }\end{array}$ \\
\hline Sleep & $5 \mu \mathrm{W}-100 \mu \mathrm{W}$ & $>98 \%$ & $\begin{array}{l}\text { Timing/memory } \\
\text { retention }\end{array}$ \\
\hline
\end{tabular}

Given a low-power node with an average sleep state of 80 microwatts $(\mu \mathrm{W})$ and receive-poll-transmit cycle of five to 10 times per hour for an overall average power draw of $100 \mu \mathrm{W}$, the device would require 876 milliwatt-hours $(\mathrm{mWh})$ to run for one year. A small lithium cell with an open circuit potential of $3 \mathrm{~V}$ and a capacity of 300 milliamp-hours (mAh) would meet this goal, discounting self-discharge and high current pulse response. Packaged, this battery weighs under five grams and is a highly reliable solution (e.g., no dependence on moving parts). ${ }^{3}$ Any power generation technology must surpass this benchmark to be a competitive solution. This is the benchmark not only for density, but for shelf life as well. Lithium-sulfur primary cells have a useful shelf life of more than 10 years.

It must be noted that current alkaline battery solutions are on the order of $\$ 0.30$ for $2500 \mathrm{mAh}$ at $1.5 \mathrm{~V}$, or $\$ 1$ for roughly $10 \mathrm{Wh}$ of electricity. This seems outrageously expensive compared to the $\$ 0.10$ charged by the local utility for a kilowatthour of electricity, but it is the tax one has to pay to get electrons in a portable format. However, as technology moves from primary and secondary batteries for power to energy harvesting and storage devices, cost, when amortized over lifetime, might be somewhere between those batteries and grid power.

\section{Micropower Generation}

Energy scavenging refers to environments where the ambient sources are unknown or highly irregular, whereas energy harvesting refers to situations where the ambient energy sources are well characterized and regular. Table II provides some examples of the differences. Just as crop harvesting provides a better foundation for human networks than hunting and gathering, energy harvesting provides a better backbone for wireless sensor networks than scavenging. For WSN applications addressing issues of grid power generation and distribution, most of the micropower work falls in the harvesting domain (which generally makes life easier for the system integrator), although some aspects, such as occupancy awareness (person tracking), might benefit from scavenging methodologies.

Table II: Comparison of Energy Harvesting and Scavenging.

\begin{tabular}{|l|c|c|}
\hline & Scavenging & Harvesting \\
\hline Photonic & Interior lighting & Diurnal solar cycles \\
\hline Thermal & Forest fires & Furnace covers \\
\hline Mechanical & Foot traffic & Motors, ductwork \\
\hline
\end{tabular}

\section{Photonic Methods}

The cost-per-area considerations for photovoltaics are secondary, as most nodes have at most a few square centimeters of exposed surface. This makes multiple-bandgap systems very attractive, particularly for energy scavenging scenarios. However, organic $^{4}$ and copper indium gallium selenide (CIGS) ${ }^{5}$ systems have demonstrated flexibility and durability innate to their design with minimal packaging. The article by Ginley et al. in this issue expands on these technologies. For "macro nodes," solar has been demonstrated in WSN applications. ${ }^{6}$ As WSN nodes approach one cubic centimeter, it might be difficult to provide the $100 \mu \mathrm{W}$ needed to keep a node in a minimum sleep state from photovoltaic techniques without secondary power. An optimistic estimate is that the sun provides roughly $100 \mathrm{~mW} / \mathrm{cm}^{2}$ incident on Earth at noon on a bright day, which can be taken as the maximum amount of harvestable energy. Indoors, that value is at least 10 times smaller and is probably less than $1 \mathrm{~mW} / \mathrm{cm}^{2}$. Conversion efficiencies are at best over $40 \%$ for multiple-bandgap systems (in laboratories at the moment), and low cost silicon solar cells can provide conversion efficiencies of $12-15 \%$ right now.

\section{Mechanical Methods}

Mechanical energy harvesting and scavenging have been widely demonstrated with lead zirconia titante (PZT), ${ }^{7}$ with exotic materials such as lead magnesium niobate-lead titanate (PMN$\mathrm{PT})^{8}$ and prosaic materials such as zinc oxide $(\mathrm{ZnO})^{9}$ and polyvinylidene difluoride $(\mathrm{PVDF})^{10}$ recently gaining attention. In any case, optimum power generation requires either matching the reso- 
nance of a vibrating structure or creating a system where resonance is achieved upon a discrete impact. Either approach requires piezoelectric materials - materials capable of transferring mechanical energy to electrical energy and vice versa. As this energy transference is implicit to the crystal or molecular structure of these materials, in theory, piezoelectric motors, pumps, actuators, and generators can be produced with fewer parts and simpler assemblies than conventional motorized devices.

In either case, resonance is a function of the geometry of the vibrating structure and the materials properties of the given piezoelectric material. Currently, the best available power density is roughly $250 \mu \mathrm{W} / \mathrm{cm}^{3}$ at an excitation of $250 \mathrm{mG} .{ }^{11}$ Capacitive ${ }^{12}$ and electromagnetic approaches have been studied and have merit but are outside the scope of this sidebar. Overall, the energy to be harvested mechanically is limited by the kinetic initiators (the moving sources), and practically, only a few percent of that initial source can be converted to electricity with these methods.

Materials advances that can improve the performance of piezoelectric energy generation within the next 10 years include the following:

- Single-crystal PZT, if epitaxial, low-temperature, solutionbased processes are developed. This could improve performance markedly at acceptable cost increases.

- Improved thin-film properties. Bulk transducers have proven advantages, ${ }^{13}$ but thin films are better suited to microfabrication and are generally easier to integrate into standard complementary metal-oxide-semiconductor (CMOS) technologies. ${ }^{14}$

- Magnetostrictive techniques, which provide magnetic dampening (or "cushioning") to the given structure. This can absorb energy, but will also increase the service life of generators ${ }^{15}$ by optimizing strain across the active surface.

As nanomaterials become commercially viable in the next 20 years, they seek to build upon the benefits of single-crystal films. Multimode resonance (structures capable of being excited by more than one frequency) or true "continuous" impact solutions (structures that produce electricity regardless of the driving frequency) can be achieved in industrial practice as they have been in the laboratory. ${ }^{9}$

Overall, materials properties are secondary to frequency dependence. Fixed-mode generators have been demonstrated, ${ }^{16}$ as have passively tuned systems. ${ }^{17}$ What is unclear is whether "actively tuned" systems that change to seek resonance automatically can ever generate more power than they use to tune to an ideal frequency. These systems would most likely require a large secondary battery, and might be able to "self-tune" only infrequently. Multimode designs, essentially xylophone-like arrays of fixed-frequency generators, have been modeled, ${ }^{18}$ but these devices trade significant power density for the extended frequency response. Finally, coupling coefficients, which are parameters describing how well the mechanical energy of the system translates to strain between the atoms of a piezoelectric material, must be considered for each individual application.

\section{Thermal Methods}

Conventional $\mathrm{Bi}_{2} \mathrm{Te}_{3}$ thermoelectric devices have been applied to wireless sensor nodes. ${ }^{19}$ As for photovoltaics, thermoelectric device requirements for micropower are nearly identical to those of their larger counterparts in terms of materials. Also similar is the constraint of a minimal footprint. For very small devices, it might be difficult to achieve the necessary temperature gradient. The sidebar by Tritt et al. in the article by Ginley et al. in this issue, discusses materials trends in thermoelectric development, as well as metrics for thermoelectric efficiency.
Overall, a balance must be struck between the leg area of thermoelectrics, which determines current, and the number of legs in series, which determines potential. If this balance is met, dc-dc conversion might be unnecessary, which would allow more volume for energy generation and improve the overall powertrain efficiency. Of course, the maximum energy that can be harvested is capped by the available gradient. Industrial plants provide perhaps $30 \mathrm{~K} / \mathrm{cm}$ effectively.

\section{On-Chip Secondary Batteries}

Because of the generally low duty cycles (ratio of time in the "on" state to time in the "off" state) of wireless sensor networks, energy density and cycle life requirements take precedence over power density. Thin-film batteries have shown promise for the past decade, ${ }^{20}$ but low capacities per area and high processing temperatures are still engineering obstacles to be overcome. Thick-film approaches will improve capacity, but a compatible, effective solid-polymer electrolyte has yet to be deployed commercially. ${ }^{21,22}$ Although liquid-phase electrolytes are an option for wireless sensor nodes, the packaging costs and environmental constraints required are generally prohibitive.

\section{Conclusion}

The continued development of micropower materials is critical to enabling self-sufficient wireless sensor nodes and networks. This development must be informed by device needs, size limitations, and manufacturing cost constraints. No single power source provides a "magic bullet" solution; thus, device makers will need a variety of materials and mechanisms to ensure that these nodes meet the application targets across varying environments.

\section{References}

1. J.M. Kahn, R.H. Katz, K.S.J. Pister, Electronics Research Laboratory Research Summary (Electronics Research Laboratory, University of California-Berkeley, 1999).

2. J. Polastre, R. Szewczyk, C. Sharp, 2006; http://www.sentilla.com (accessed January 2008).

3. Renata Batteries, CR2430 3V Lithium Battery Technical Data Sheet (Renata Batteries, 2007; www.renata.com/pdf/3vlithium/DBCR2430.04.pdf) (accessed January 2008).

4. M. Gratzel, MRS Bull. 30 (1), 23 (2005).

5. N.G. Dherea, R.G. Dhere, J. Vac. Sci. Technol. A 23 (7-8), 1208 (2005).

6. J. Xiofan, J. Polastre, D. Culler, IPSN/SPOTS 2005 (2005).

7. S. Roundy, P.K. Wright, Smart Mater. Struct. 13 (10), 1131 (2004).

8. K.L. Ren, Y.M. Liu, X.C. Geng, H.F. Hofmann, Q.M.M. Zhang, IEEE Trans. Ultrason. Ferroelectr. Freq. Control 53 (3), 631 (2006).

9. Z.L. Wang, Mater. Today 10 (5), 20 (2007).

10. C.S. Lee, J. Joo, S. Han, S.K. Koh, Sens. Actuators A, Phys. 121 (6), 373 (2005).

11. S. Roundy, D. Steingart, L. Frechette, P. Wright, J. Rabaey, Wireless Sens. Netw., Proc. 2920, 1 (2004).

12. P.D. Mitcheson, P. Miao, B.H. Stark, E.M. Yeatman, A.S. Holmes, T.C Green Sens. Actuators A, Phys. 115, 523 (2004).

13. S.P. Beeby, N. Ross, N.M. White, Electron. Lett. 35, 2060 (Nov 11, 1999). 14. M.H.M. Zai, A. Akiba, H. Goto, M. Matsumoto, E.M. Yeatman, Thin Solid Films 394, 97 (Aug 15, 2001).

15. J.K. Huang, D. Bono, R.C. O'Handley, Sens. Lett. 5 (3), 105 (2007).

16. S. Roundy, J. Intell. Mater. Syst. Struct. 16 (10), 809 (2005).

17. E.S. Leland, P.K. Wright, Smart Mater. Struct. 15 (10), 1413 (2006)

18. S.M. Sharuz, Mechatronics 16, 523 (2006).

19. J.W. Evans, M. Schneider, D. Steingart, D. Ziegler, P. Wright, in Light Metals 2005, H. Kvande, Ed. (TMS, Warrendale, PA, 2005), p. 407.

20. J.B. Bates, N.J. Dudney, B. Neudecker, A. Ueda, C.D. Evans, Solid State Ionics 135, 33 (2000).

21. D. Steingart, C. Ho, J. Salminen, J.W. Evans, P.W. Wright, IEEE Polytronic 2007: 6th International IEEE Conference on Polymers and Adhesives in Microelectronics and Photonics, Odaiba, Tokyo, Japan, 16-18 January 2007. 22. M. Ollinger, H. Kim, T. Sutto, A. Pique, Appl. Surf. Sci. 252, 8212 (September 30, 2006). 


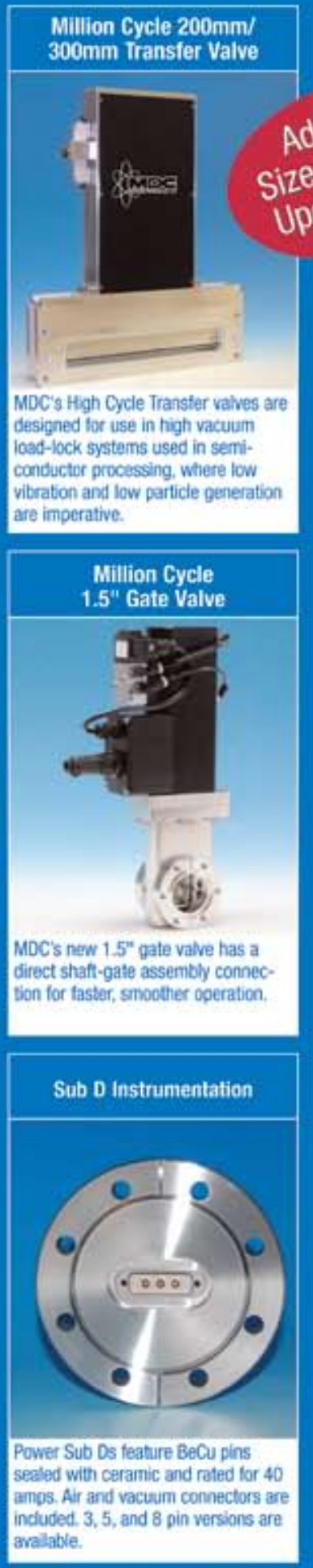

\section{Vacuum Components for} additional upon Request Energy Materials Research Applications

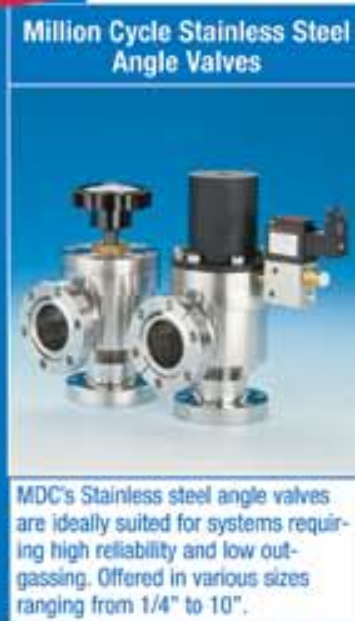

Designed for Performance, reliability and value. MDC offers a full line of HV \& UHV vacuum valves manufactured to accommodate all of your Materials Research, R\&D, Thin-film and semiconductor processing needs. MDC's valve line includes gate, angle, in-line, butterfly, leak and all metal valves. Call or visit us online for more information on MDC's high performance million cycle valves.
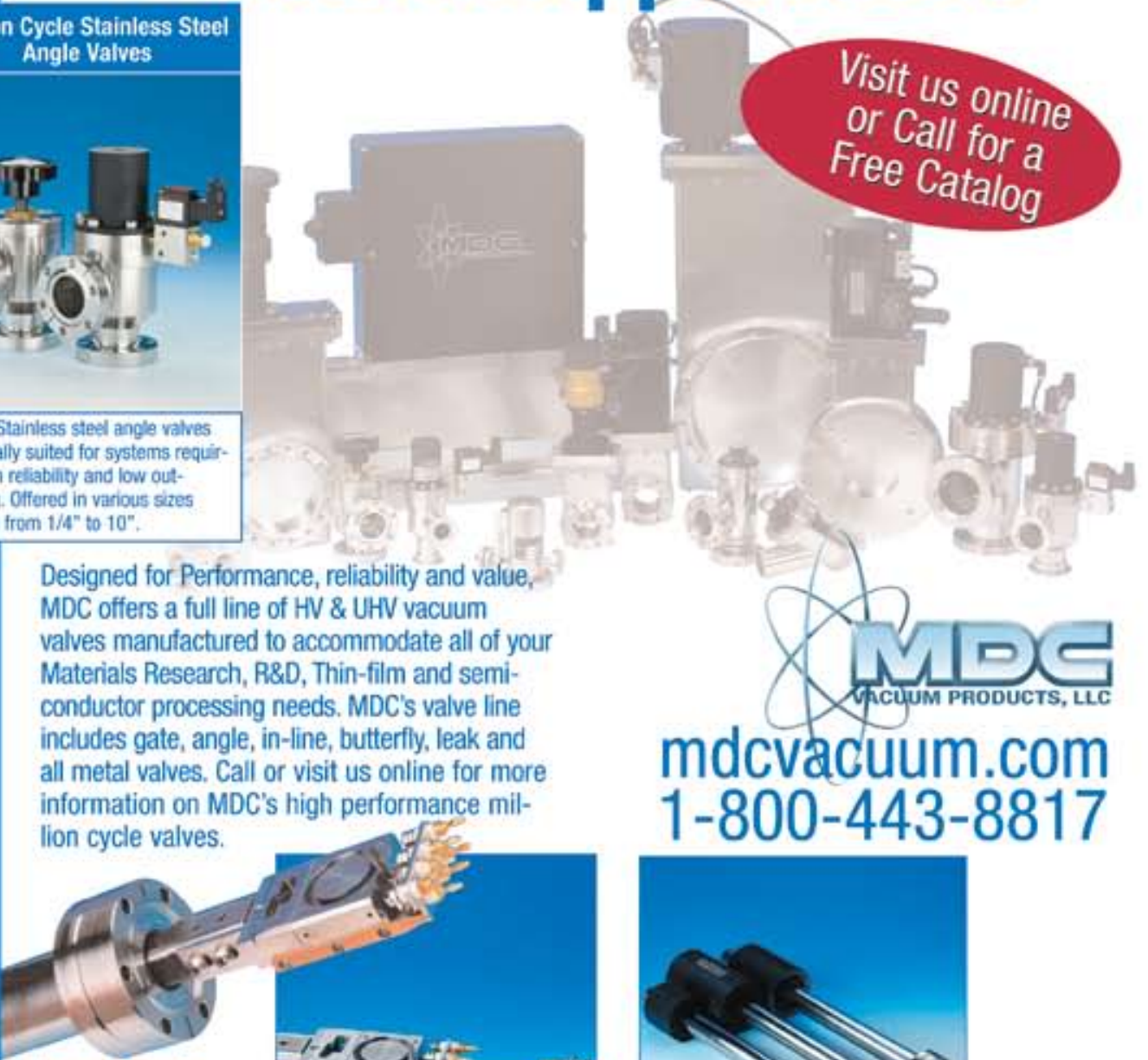

Advanced Sample Manipulation System
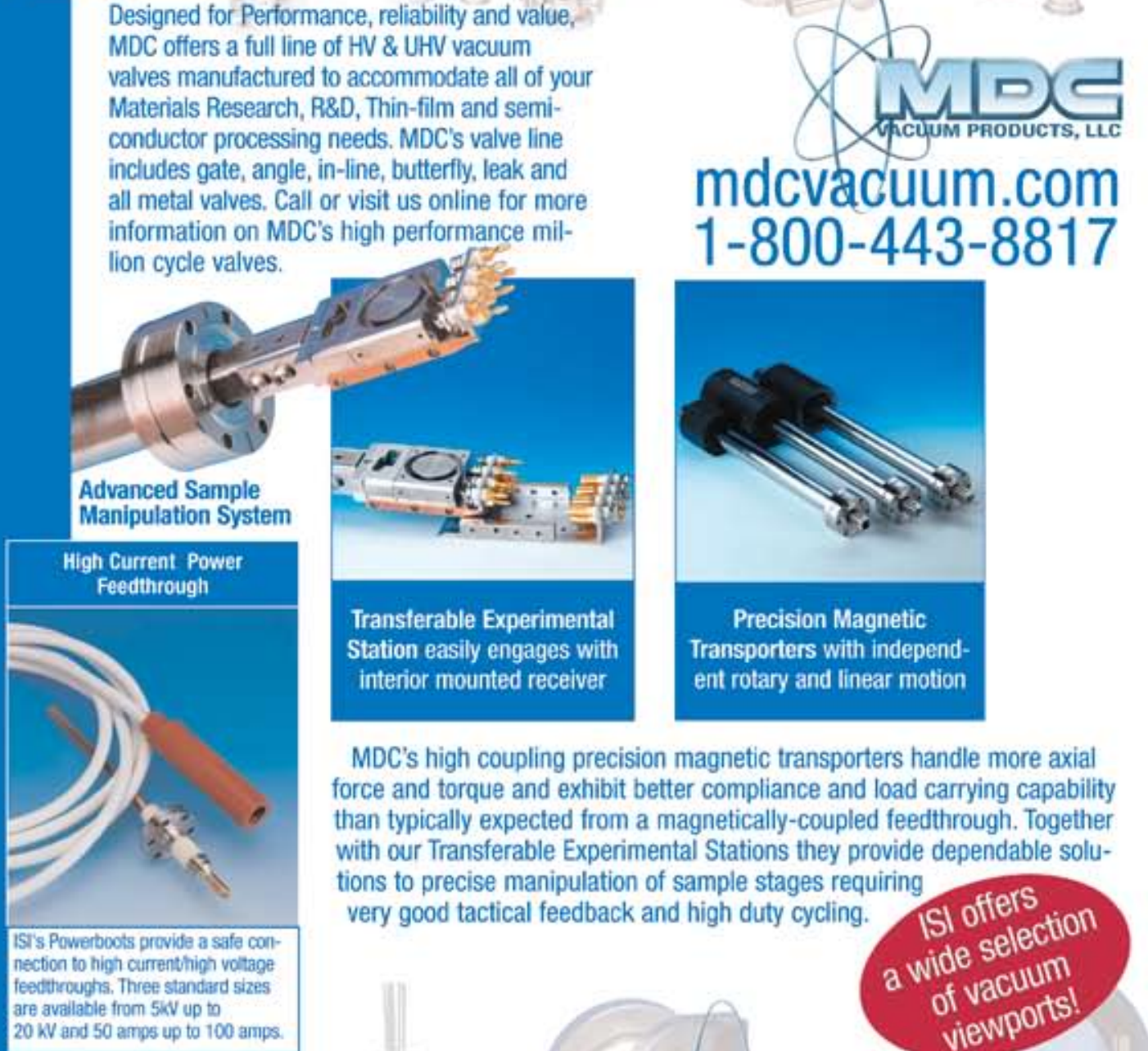
$1-800-443-8817$

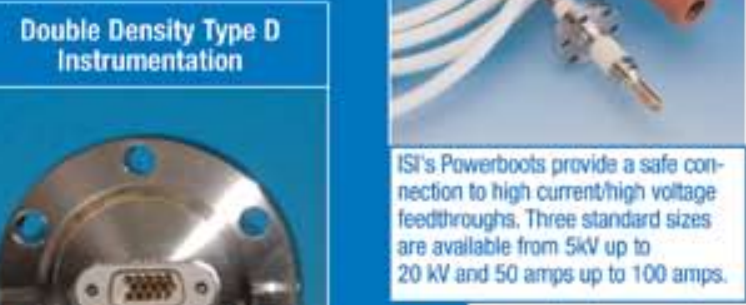

MDC's high coupling precision magnetic transporters handle more axial force and torque and exhibit better compliance and load carrying capability than typically expected from a magnetically-coupled feedthrough. Together with our Transferable Experimental Stations they provide dependable solutions to precise manipulation of sample stages requiring very good tactical feedback and high duty cycling.

ISI features the most extensive product offering in the vacuum feedthrough industry today. From High-Powered to RF applications, ISI can fill all your needs for custom designed products or standard catalog items. Call or visit us online for more information on Insulator

Seal's Feedthrough product line.

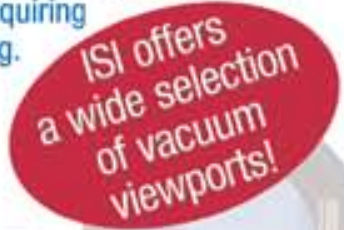

